\title{
Heat conduction and long-range spatial correlation in 1D models
}

\author{
Xin Zhou and Mitsumasa Iwamoto \\ Department of Physical Electronics, Tokyo Institute of Technology, O-okayama 2-12-1, Meguro-ku, Tokyo 152-8552, Japan
}

(November 3, 2018)

\begin{abstract}
Heat conduction in one-dimensional (1D) systems is studied based on an analytical S-matrix method, which is developed in the mesoscopic electronic transport theory and molecular dynamic (MD) simulations. It is found that heat conduction in these systems is related to spatial correlation of particle motions. Randomizations of scatterers is found to break the correlation, hence results in normal thermal conduction. Our MD simulations are in agreement with the theoretical expectations. The results are useful for an understanding of the relation between heat conduction and dynamic instablities or other random behavior in 1D systems.
\end{abstract}

PACS numbers: 44.10.+i, 05.45.-a, 05.60.-k, 05.70.Ln

\section{INTRODUCTION}

The study of heat conduction in one-dimensional (1D) systems is an interesting subject in the context of nonequilibrium statistical physics, which has been attracting much attention in recent years [1]. Most works aim at understanding the dynamic properties of heat transport in 1D systems [2-10]. Although many modelling systems have been carefully studied and their thermal conductivities and temperature profiles calculated, the key dynamic properties of normal conduction is still unknown. Generally, normal heat conduction is specified by a diffuse-type motion, hence the investigation of random behavior of systems is necessary. It is well known that chaos can generate the needed random behavior in some systems. For example, thermal conductivity is characterized normal in the ding-a-dong model [2] and the Lorentz gas channels [6], due to their dynamic instability. However heat conduction is still considered abnormal in other systems, such as Fermi-Pasta-Ulam (FPU) model [3] in spite of their dynamic instability. Recently, $\mathrm{Li}$ et al. [9] studied three models with the same zeroLyapunov exponents and found that heat conduction can be either normal or abnormal depending on details of the models. However they could not reach the conclusion that there is direct connection between dynamic instability and normal heat conduction. From these studies, the most important and general conclusion is that the momentum conservation systems with non-zero presure have anomalous conductivity, whereas, anomalous conductivity does not always imply momentum conservation. Prosen and Campbell [11] were the first to prove this conclusion. Recently, Narayan and Ramaswamy [12] criticized that the original proof is questionable, but there is no strong objection to deny the conclusion. The general theoretical result will explain the abnormal heat transport found in FPU model, and may clarify the recent confused results obtained by numerical works in some models, such as diatomic Toda model. Heat conduction in the Toda model was thought as normal by Jackson et al. [13] and by Garrido et al. [14], but was against the numerical results by Hatano [5], Dhar [8], Savin et al. [15] and Grassberger et al. [10]. This indicates that it is difficult to get definite conclusions about the macroscopic transport properties of nonlinear systems by merely using short-time numerical simulations in small systems.

In this paper, based on both theoretical and numerical methods, we try to explain the required dynamic characteristics that guarantee normal heat conduction in 1D systems. By considering a 1D model with $N$ scatterers and noninteracting classic particles, we find that the classic model can be theoretically treated using S-matrix theory [16] developed in the mesoscopic electronic transport theory (METT). In METT, if the scattering is random, electronic transport will be incoherent, electronic phase correlation at the two ends of a $1 \mathrm{D}$ chain will be absent, and eventually the Ohm's law is observed. Similarly, in our model, defining a 'phase' correlation, we find that normal heat conduction is characterized by the broken of phase correlation, where the randomization of the scatterers makes a main contribution. This conclusion is supported by some principle considerations and previous related researches and can be applied to more general systems. Our result indicates that a sufficient random characteristic of $1 \mathrm{D}$ systems is necessary to obtain normal heat conduction. The random may be ensured by dynamic instablity, randomization of scatterers or other matters and could destroy long range correlations of particle motion along the 1D chains. Therefore, dynamic instability is not a necessary condition to guarantee normal transport if other random effects exist.

\section{THEORY}

\section{A. Model and Scattering Theory}

Consider a 1D classical chain with $N$ scatterers $s_{k}$, $(k=1, \cdots, N)$ and non-interactive particles, which are elastically transmitted or reflected at these scatterers, we place this chain between two heat baths. Without loss of generality, the length of chain $(L)$ is set as $N$. If the transmission coefficient $\left(t_{k}\right)$ of each scatterer is a number between 0 and 1 , it is a typical random walk pro- 
cess, hence heat conduction is normal and independent of the kind of heat baths. However, here we suppose that scatterers periodicaly turn on or off in time, so particles will completely transmit through a scatterer in some time ranges, but reflect at the scatterer in the other time ranges. So the transmission coefficients are function of time $\phi,\left(t_{k}(\phi)\right)$. It is a typically deterministic system. Here, we set the period $t_{k}(\phi)$ to 1 and the average transmit coefficient to $1 / 2$. We suppose that there are different initial time shifts $\delta_{k}$ for different scatterers, then we have $t_{k}(\phi)=t\left(\phi-\delta_{k}\right)$, and

$$
t(\phi)= \begin{cases}1, & 0 \leq \phi<1 / 2 \\ 0, & 1 / 2 \leq \phi<1 .\end{cases}
$$

The term $\phi$ as well as $\delta_{k}$ are thought as 'phases'. Another parameter of the $k$ th scatterer is its positions $x_{k}$. $\left\{\delta_{k}\right\}$ and $\left\{x_{k}\right\}$ will determine the properties of the model. Actually, the model is very similar to the Lorentz channel model [6] or the Ehrenfest gas channel model [9].

We define the average of the particle current density $J(x, v, t)$ on the model,

$$
j(x, v, \phi ; n)=\frac{1}{M} \sum_{m=0}^{M-1} J(x, v, \phi+m+n),
$$

where $n, m$ are integer numbers, $M$ is a large integer number, and $0 \leq \phi<1$. In steady state, $j(x, v, \phi ; n)$ is independent of $n$, and is noted as $j(x, v, \phi)$. Due to the particle current conservation, if $x$ is between two nearest neighbor scatterers, $x_{k}$ and $x_{k+1}, J(x, v, t)$ is written as $J_{v}(t-x / v)$. Hence we have,

$$
\begin{array}{r}
j(x, v, \phi)=\sum_{m} j_{m}^{(k)}(v) \exp \{2 m \pi i(x / v-\phi)\}, \\
\text { if } \quad x_{k}<x<x_{k+1} .
\end{array}
$$

The heat current density $J_{u}(x)$ and temperature profile $\mathcal{T}(x)$ can be writen as,

$$
J_{u}(x)=\frac{1}{2} \int_{0}^{\infty} d v v^{2}\left[j_{0}^{(k)}(v)-j_{0}^{(k)}(-v)\right],
$$

and

$$
\mathcal{T}(x)=\frac{\int_{0}^{\infty} d v v\left[j_{0}^{(k)}(v)+j_{0}^{(k)}(-v)\right]}{\int_{0}^{\infty} d v v^{-1}\left[j_{0}^{(k)}(v)+j_{0}^{(k)}(-v)\right]},
$$

respectively, while $x_{k}<x<x_{k+1}$.

Based on the properties of scatterers, we easily obtain the following scattering formula,

$$
\left(\begin{array}{c}
\hat{j}^{(k)}(v) \\
\hat{j}^{(k-1)}(-v)
\end{array}\right)=\left(\begin{array}{cc}
\hat{t}^{(k)}(v) & \hat{r}^{\prime(k)}(v) \\
\hat{r}^{(k)}(v) & \hat{t}^{(k)}(v)
\end{array}\right)\left(\begin{array}{c}
\hat{j}^{(k-1)}(v) \\
\hat{j}^{(k)}(-v)
\end{array}\right)
$$

where $\hat{t}, \hat{r}, \hat{t}^{\prime(k)}$, and $\hat{r}^{\prime(k)}$ are nothing but S-matrix elements. Since this is a multi-mode scattering problem, each S-matrix element is an infinite dimensional matrix.
For example, the element $t_{m n}^{(k)}$ of $\hat{t}^{(k)}$ means a coefficient which the mode $m$ at the left transmit the scatterer $k$ to mode $n$ at the right,

$$
t_{m n}^{(k)}(v)=t^{(m-n)} \exp \left\{-2 \pi i(m-n)\left(x_{k} / v-\delta_{k}\right)\right\},
$$

where $t^{(p)}=\int_{0}^{1} d \phi t(\phi) e^{2 p \pi i \phi}$, is the $p$ th Fourier's expanded coefficient of transmission function $t(\phi)$. Similarly, other matrixes can be writen as,

$$
\begin{aligned}
r_{m n}^{(k)}(v) & =r_{k}^{(m-n)} \exp \left[-2 \pi i(m+n) x_{k} / v\right], \\
t_{m n}^{\prime(k)}(v) & =t^{(m-n)} \exp \left[-2 \pi i(m-n)\left(x_{k} /(-v)-\delta_{k}\right)\right], \\
r_{m n}^{\prime(k)}(v) & =r_{k}^{(m-n)} \exp \left[-2 \pi i(m+n) x_{k} /(-v)\right],
\end{aligned}
$$

where $r_{k}^{(p)}=\left(\delta_{p, 0}-t^{(p)}\right) \exp \left(2 p \pi i \delta_{k}\right)$.

For whole $N$ scatterers, we have,

$$
\left(\begin{array}{c}
\hat{j}^{(N)}(v) \\
\hat{j}^{(0)}(-v)
\end{array}\right)=\left(\begin{array}{cc}
\hat{T}(v) & \hat{R}^{\prime}(v) \\
\hat{R}(v) & \hat{T}^{\prime}(v)
\end{array}\right)\left(\begin{array}{c}
\hat{j}^{(0)}(v) \\
\hat{j}^{(N)}(-v)
\end{array}\right)
$$

where $\hat{T}, \hat{R}, \hat{T}^{\prime}$ and $\hat{R}^{\prime}$ are whole transmitting and reflecting matrix of all scatterers from left to right and from right to left, respectively. $\hat{j}^{(0)}$ and $\hat{j}^{(N)}$ correspond to the current densities at the left and right ends, respectively.

Since the parameters of the system $\left(x_{k}\right.$ and $\left.\delta_{k}\right)$ determine the S-matrix, we study heat conduction of models with different parameters. Without loss of generality, we set

$$
\begin{aligned}
\delta_{k} & =c * R, \\
x_{k} & =k-0.5+d *(R-0.5),
\end{aligned}
$$

where $R$ is a random number uniformly distributed between 0 and $1, c$ and $d$ are the magnitude of disorder in scattering phases and positions, respectively. If both $c$ and $d$ equal to zero, it is a periodic scattering system, otherwise, it is a disordered system. For the latter, we can consider two kinds of different disordered systems: (1) the dynamic random system (DRS), where $x_{k}$ or $\delta_{k}$ are random in time; (2) the static random system(SRS), where random $x_{k}$ or $\delta_{k}$ are fixed rather than depending of time in each realizations of system. We are interested in the average properties of many realizations. DRS and SRS correspond to the phonon scattering and impure scattering in electronic transport, respectively. Obviously, the disorder of $x_{k}$ or $\delta_{k}$ will induce some random phases in the S-matrix, it may affect the transport property of the system.

The combining S-matrices of $N$ scatterers can be easily written according to the well known rules generated in METT: it is written as a summation of many terms, where each term is depicted by a 'Feynman path' [16]. For example, the combining transmitting matrix of any two parts of scattering $s^{(1)}$ and $s^{(2)}$ is,

$$
\begin{aligned}
\hat{t}(12) & =\hat{t}^{(2)}\left[I-\hat{r}^{(1)} \hat{r}^{(2)}\right]^{-1} \hat{t}^{(1)}, \\
& =\hat{t}^{(2)}\left[I+\hat{r}^{(1)} \hat{r}^{(2)}+\cdots\right] \hat{t}^{(1)} .
\end{aligned}
$$


Generally, we have $T_{m n}(N)=\sum_{P} A_{P}$, where each $A_{P}$ is a complex number contributed from path $P$ which starts from mode $n$ at the left end, to mode $m$ at the right end. If $t_{k}(\phi)$ of each scatterer is independent of time $\phi$, then there is only one mode, $T=\sum_{P_{0}} A_{P_{0}}$, where $P_{0}$ are all the 'Feynman' paths with mode 0 for all scatterers. We easily obtain,

$$
T(N)=\left(\sum \frac{1-t_{i}}{t_{i}}+1\right)^{-1} \sim \frac{t}{N(1-t)+t} .
$$

While $N \rightarrow \infty$, we have $T(N) \sim 1 / N$, satisfying the Fourier's law, which is the expectation of the random walk process.

\section{B. Heat Baths}

Before going on to the study of the transport properties of the model, we analyze the effects of heat baths. Here, the temperatures of the left and right heat baths are noted as $T_{1}$ and $T_{2}$, respectively. Many kinds of heat baths can be selected. For example, we can choose complete-reflecting heat baths: as a particle hits a heat bath, it will be reflected with a velocity distribution $P_{T}(v)$, where $T$ is the temperature of the heat bath. $P_{T}(v)$ can be of the Maxwellian distribution, $P_{T}(v)=$ $v / T \exp \left(-v^{2} / 2 T\right)$, or of a single velocity distribution, $P_{T}(v)=\delta(v-\sqrt{T})$, or other forms. In this case, we have completely reflecting conditions at the boundaries:

$$
\begin{aligned}
j_{m}^{l}(v) & =J_{m}^{l} P_{T_{1}}(v) \\
j_{m}^{r}(-v) & =J_{m}^{r} P_{T_{2}}(v),
\end{aligned}
$$

where $v$ is larger than $0, j_{m}^{l}(v) / j_{m}^{r}(-v)$ corresponds to the currents entered into the 1D system from the left/right heat bath $(x=0 / L)$, which is a different phase from $j_{m}^{0}(v) / j_{m}^{N}(-v)$ which are used in eq. (9). $J_{m}^{l}=\int_{0}^{\infty} d v j_{m}^{l}(-v)$ and $J_{m}^{r}=\int_{0}^{\infty} d v j_{m}^{r}(v)$. Actually, $J_{m}^{l}$ and $J_{m}^{r}$ are the Fourier expanded coefficients of the current densities $J^{l}(\phi)$ (at the left end, $x=0$ ) and $J^{r}(\phi)$ (at the right end, $x=L$ ) of $1 \mathrm{D}$ chain, respectively. From eq. (9), we easily obtain,

$$
\left(\begin{array}{c}
\hat{j}_{m}^{r}(v) \\
\hat{j}_{m}^{l}(-v)
\end{array}\right)=\left(\begin{array}{cc}
\tilde{T}_{m n}(v) & \tilde{R}_{m n}^{\prime}(v) \\
R_{m n}(v) & \tilde{T}_{m n}^{\prime}(v)
\end{array}\right)\left(\begin{array}{c}
\hat{j}_{n}^{l}(v) \\
\hat{j}_{n}^{r}(-v)
\end{array}\right)
$$

where there are some phase differences between the matrix elements and the original S-matrix elements in eq. (9),

$$
\begin{aligned}
& \tilde{T}_{m n}(v)=\exp (2 m \pi i L / v) T_{m n}(v) \\
& \tilde{T}_{m n}^{\prime}(v)=T_{m n}^{\prime} \exp (2 n \pi i L / v) \\
& \tilde{R}_{m n}^{\prime}(v)=\exp (2 m \pi i L / v) R_{m n}^{\prime} \exp (2 n \pi i L / v)
\end{aligned}
$$

Then, we know $J_{m}^{l}$ and $J_{m}^{r}$ are not arbitrary, but satisfying the following equation,

$$
\left(\begin{array}{cc}
<R_{m n}>_{1}-\delta_{m n} & <\tilde{T}_{m n}^{\prime}>_{2} \\
<\tilde{T}_{m n}>_{1} & <\tilde{R}_{m n}^{\prime}>_{2}-\delta_{m n}
\end{array}\right)\left(\begin{array}{c}
J_{n}^{l} \\
J_{n}^{r}
\end{array}\right)=0
$$

where $<f_{m n}>_{i},(i=1$, or 2$)$, means the average value of $f_{m n}(v)$ under the velocity distribution function $P_{T_{i}}(v)$. The physical meaning of eq.(18) will be easily understood. In fact, we can treat the right boundary as $(N+1)$ th scatterer ( complete reflecting), $J_{m}^{l}$ must satisfy the linear equation, $\left(R^{N r}-I\right) J^{l}=0$, where $R^{N r}$ is the total reflecting matrix of all $N$ scatterers as well as the right heat bath. From Eq. (18), we have

$R^{N r}=<R>_{1}+<\tilde{T}^{\prime}>_{2}\left[I-<\tilde{R}^{\prime}>_{2}\right]^{-1}<\tilde{T}>_{1}$.

Since

$$
[I-R]^{-1}=I+R+R R+\cdots
$$

we find that the result is nothing but the summation of 'Feynman path'. Similarly, we also have the formula of $R^{l N}$ and equation $\left(R^{l N}-I\right) J^{r}=0$. If there is only one linear independent solution of the equation, we will get a unique steady state, (a free constant $J_{0}^{l}$ is decided by the particle density and average temperature of system).

However, the asymptotic $N$ dependence of the heat current is only determined by the $N$ dependence of transmission coefficient $|\hat{T}|$, which is independent of the details of heat baths. In this paper, we only consider a simpler case, replacing with the completely reflecting heat baths: particles uniformly enters into the system from two heat baths at time $(\phi)$ with the single velocity distribution $\delta(v-\sqrt{T})$, therefore the heat current is simply proportional to the transmission coefficient $T_{00}$. When calculating $\mathcal{T}(x)$, we choose another uniform incoming current from the right heat bath at the same time, making the total particle current zero.

\section{Phase Correlation}

It is well established that, if the scattering is random, the contributions from different Feynman paths are incoherent in electronic transport, therefore, we can find ohm's law and normal electric conductivity. But if there are some long range correlations between scatterers, the electronic phase-relaxation length may be larger than the length of the system, the transport will be coherent and we can find anomalous electric conductivity. So the existence of the phase correlation between electrons at the two ends can be used to judge whether the transport be coherent or not, hence whether the conductivity be abnormal or normal. Comparing the results, we expect a similar relationship between heat conduction and a long range correlation of particles in $1 \mathrm{D}$ systems. We also expect the random charactaristic of scatterers to be responsible for the correlations.

For any incoming particle current from the left heat bath, $J_{+}^{l}(\phi)$, we have the transmit current density at 
the right boundary $J_{+}^{r}(\psi)=\int T(\psi, \phi) J_{+}^{l}(\phi) d \phi$, where $T(\psi, \phi)$ is the transmit function,

$$
T(\psi, \phi)=\sum_{m n} \exp (-2 m \pi i \psi) T_{m n} \exp (2 n \pi i \phi)
$$

Similarly, the reflecting function is $R(\psi, \phi)$. Since for any $\phi, \int[T(\psi, \phi)+R(\psi, \phi)] d \psi=1$, so $R_{0 n}=\delta_{0 n}-T_{0 n}$.

The probability that we observe a current with phase $\psi$ at the right end and a current with $\phi$ at the left end is $W_{2}(\psi, L ; \phi, 0)=T(\psi, \phi) J_{+}^{l}(\phi)$. We define a normalized two-point current distribution function as $f_{2}(\psi, \phi)=$ $W_{2}(\psi, N ; \phi, 0) / A$, where $A$ is a normalized constant,

$$
A=\int W_{2}(\psi, L ; \phi, 0) d \phi d \psi=\int J_{+}^{r}(\psi) d \psi .
$$

The left and right normalized distributions are

$$
f_{l}(\phi)=\int f_{2}(\psi, \phi) d \psi
$$

and

$$
f_{r}(\psi)=\int f_{2}(\psi, \phi) d \phi
$$

respectively.

Based on these distributions, we define a motion correlation of particles at two ends of the 1D chain as,

$$
D=\frac{\langle\phi \psi\rangle_{2}-\langle\phi\rangle_{l}\langle\psi\rangle_{r}}{\sigma_{l} \sigma_{r}},
$$

where $\langle\cdots\rangle_{i}(i=2, l$ or $r)$ means the average value under the distribution functions $f_{2}(\psi, \phi), f_{r}(\psi)$ and $f_{l}(\phi)$, respectively. $\sigma_{l / r}=\sqrt{\left(\left\langle\phi^{2}\right\rangle_{l / r}-\langle\phi\rangle_{l / r}^{2}\right)}$ is the width of the current distribution at the left/right end. Hence, $D=0$ implies the broken of the correlation and $T(\psi, \phi)=$ $F(\psi) G(\phi)$ (or $T_{m n}=F_{m} G_{n}$ ). Here, $D$ corresponds to the electronic phase correlation at both ends of $1 \mathrm{D}$ electronic transport system, and the particle velocity play the role of wavevector of electron.

\section{Theoretical Results}

Based on the rules of the 'Feynman' paths on scattering theory, the transmission matrix $T_{m n}$ can be writen as,

$$
T_{m n}=\sum_{p q} t_{m p}^{(N)} t_{q n}^{(1)} B_{p q}(N)
$$

where $t^{(N)}$ and $t^{(1)}$ are the transmission matrix of the scatterer $\mathbf{N}$ and the scatterer $\mathbf{1}$, respectively. $B_{p q}(N)$ is the sum of all 'Feynman' paths which start from mode $q$ of the scatterer $\mathbf{1}$, end to mode $p$ of the scatterer $\mathbf{N}$. Expanding $B_{p q}(N)$ in large $N$, the lead term is noted as $\alpha_{p q} / N^{\gamma}$, therefore we have the first result that the asymptotic $N$ dependence of $T_{m n}$ is independent on $m$ and $n$,

$$
T_{m n}=T(N) h_{m n},
$$

where $T(N) \sim 1 / N^{\gamma}$, is a simple notation of $T_{00}$ and $h_{m n}$ is independent of $N$. If the correlation is absent, $T_{m n}=T(N) f_{m} g_{n}$.

For DRS, due to the random time-dependent positions/phase of the scatterers, the contribution from different Feynman paths are not coherent with each other, so they can be first averaged in time, then be summed up. This indicates that only 0-mode contribute to the total transmission coefficient, so the heat conduction shall be normal. We also easily know that the correlation is absent in DRS due to the incoherent Feynman contribution.

\section{NUMERICAL SIMULATION}

In this section, by using a uniform inputing current with single velocity $v_{1}=\sqrt{T_{1}}$ from the left end, we numerically simulate the transmit coefficient $T(N)$, the distribution function $f_{r}(\psi), f_{l}(\phi)$ and the temperature profile $\mathcal{T}(x)$ in different systems. First, we find that both $f_{r}(\psi)$ and $f_{l}(\psi)$ are independent of $N$ for all models including the periodic scattering system, DRS and SRS with the same disordered magnitude $(c$ and $d)$. The results verify very well our theoretical expectations in eq. (27) and indicate that $h_{m n}$ is only dependent on the disordered magnitude. As $c$ (or $d$ ) increases, $f_{r}(\psi)$ and $f_{l}(\phi)$ are flatter, but they are not uniform even though the disordered magnitudes arrive at their maximum value (1). For example, the results in DRS are shown in Fig. 1 , where $J^{r}(\psi)$ is the unnormalized current distribution function at the right end. Then, we show the temperature profiles $\mathcal{T}(X / N)$ of DRS in Fig. 2(a), which are found to being independent on $N$ but dependent on the disorder magnitude $c$ (or $d$ ). It indicates that $d \mathcal{T} / d X$ is proportional to $1 / N$. The heat current $J_{u} \sim 1 / N^{\gamma}$ is shown in Fig. 2(b), and fitted $\gamma \approx 0.9989 \pm 0.0045$. Therefore the heat conduction is normal in DRS with disordered scattering phases, which verifies our theoretical expectation. In this case, our obtained spatial correlation $D$ is very small but with the same order statistic error. For a better correlation estimation, we used the non-uniform input currents $J_{+}^{l}(\phi)$ with different distributions and found the right-end distribution functions $f^{r}(\psi)$ independent of the selection of $J_{+}^{l}(\phi)$ (not shown). This indicates that the transmission function $T(\psi, \phi)$ can be writen as $F(\psi) G(\phi)$, hence $D=0$. For DRS with disordered scattering positions, the obtained results are similar as that of DRS with disordered phases.

We also numerically simulate the heat conduction of SRS. Since the position disordered system is similar to the phase disordered system, we only show the results of the latter. The temperature profile $\mathcal{T}(x)$ and the $N$ 
dependence of $J_{u}$ in SRS are similar as that in DRS, but $\gamma \approx 1.19$ in SRS, slightly larger than 1 in DRS (seeing Fig. 3) and there is a slight difference on $\mathcal{T}(x / N)$ of systems with different $N$. The differences may be due to the limiting chain length $N$ in our simulation. For different disordered magnitude $c$, the value of $\gamma$ is found to be indistinguishable. For comparison, we simulate the heat current $J_{u}$ in a periodic scattering system, the result is also not very clear. It may be necessary to simulate them in longer chains. In Fig. 4, we show the distribution of $D$ for different disordered realizations in SRS. The average of $D$ is about zero, in agreement with our expectation which the scattering disorders induce the absence of the correlation. Other interesting result is shown in Fig. 4(b): the fluctuation of $D$ decreases as $N$ increases, $\left\langle D^{2}\right\rangle=N^{-0.42}$. It indicates that $D \rightarrow 0$ in any single disordered realization of SRS while $N \rightarrow \infty$. Contrarily, in periodic system, the correlation $D$ is not zero.

\section{DISCUSSIONS AND CONCLUSION}

We have defined a spatical correlation along the 1D chains to describe scattering (i. e. transport) characteristic in the systems and theoretically expected that randomization of scattering determineed the existence of the long range spatial correlation, therefore properties of heat conduction. For DRS, the expectation was confirmed by both S-matrix theory and molecular dynamic simulation. For SRS and periodic system, according to the obtained correlations, normal and abnormal heat conductions were expected, respectively. But our numerical simulations in some small systems could not completely clear the expectation, more simulations might be necessary. A noted fact is that our model is very similar to the Ehrenfest gas channel [9]. In the latter, the channel is quisal-1D systems with a small transverse coordinate (the height of the channel). Actually, the height corresponds to the 'phase' in our models, a similar scattering theory can be derived. For the Ehrenfest channel, the results of Li et al. [9], which heat conduction is normal if the heights or positions of the scatterers are random and it is anormalous if their heights or positions are periodic, can be easily understood from our results. In these cases, the required incoherent characteristic of motion to get normal heat conduction can be ensured by the random 'phases' and positions of scatterers, not needing additive dynamic chaos (non-zero Lyapunov Exponent). Therefore, the conditions with normal heat conduction in 1D systems are whether or not there are sufficient random to destory completely the correlation of motions along chains. The random may come from dynamic instablity, random scatterers or another sources, but no any particular random, such as chaos, is necessary. The judgement of the incoherent motions (i. e. normal transport) is whether or not the long range spatial correlations exist.

We also can consider another models, for example, an variant of ding-a-dong model, which its even-numbered particles are oscillatedly coupled each other, rather than coupled with its individual lattice site in the initial model [2]. Due to the existence of some long-wave modes in the model, the motion correlation is obviously present, we expect the thermal conductivity should be abnormal. On general 1D systems, important questions are how to define suitable correlations and judge directly the existence of the correlations from the characteristic of systems. For example, for momentum conservation systems, which thermal conductivities are anomalous if presure is not zero [11], it is worthy for connecting the conditions with existence of spatial correlations.

X. Z. was supported by the Grants-in-Aid for Scientific Research of JSPS, his current address is zhou@pe.titech.ac.jp. X. Z. thanks the help of Mr. C.-Q. Li.

[1] F. Bonetto, J. L. Lebowitz, and L. Rey-Bellet, e-print math-ph/0002052.

[2] G. Casati, et. al., Phys. Rev. Lett. 52, 1861 (1984).

[3] S. Lepri, R. Livi, and A. Politi, Phys. Rev. Lett. 78, 1896 (1997).

[4] B. Hu, B. Li and H. Zhao, Phys. Rev. E. 57, 2992 (1998).

[5] T. Hatano, Phys. Rev. E 59, R1 (1999).

[6] D. Alonso, R. Artuso, G. Casati, and I. Cuarneri, Phys. Rev. Lett. 82, 1859 (1999).

[7] O. V. Gendelman, and A. V. Savin, Phys. Rev. Lett. 84, 2381 (2000).

[8] A. Dhar, Phys. Rev. Lett. 86, 3554 (2001).

[9] B. Li, L. Wang, and B. Hu, Phys. Rev. Lett. 88, 223901 (2002).

[10] P. Grassberger, W. Nadler, and L. Yang, Phys. Rev. Lett. 89, 180601 (2002).

[11] T. Prosen, and D. K. Campbell, Phys. Rev. Lett. 84, 2857 (2000).

[12] O. Narayan, and S. Ramaswamy, Phys. Rev. Lett. 89, 200601 (2002);

[13] E. A. Jackson and A. D. Mistriotis, J. Phys.: Condens. Matter 1, 1223 (1989).

[14] P. L. Garrido, P. I. Hurtado, and B. Nadrowski, Phys. Rev. Lett. 86, 5486 (2001).

[15] A. V. Savin, G. P. Tsironis, and A. V. Zolotaryuk, Phys. Rev. Lett. 88, 154301 (2002).

[16] S. Datta, Electronic Transport in Mesoscopic Systems, Cambridge Univ. Press (1995). 
X. Zhou et al. Fig. 1
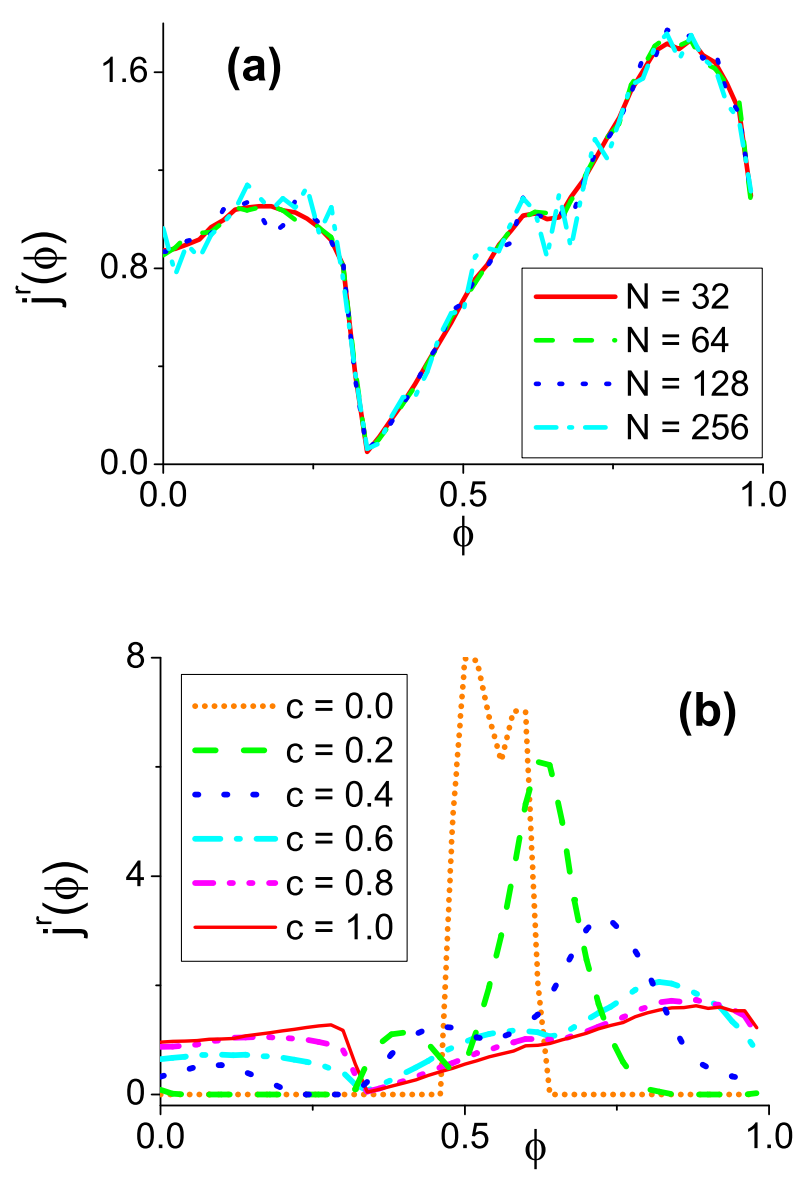

FIG. 1. The particle current distribution $J^{r}(\phi)$ at the right end in DRS with random phases: (a) $J^{r}(\phi)$ of systems with different $N$ are identified each other, where $c=0.8$. (b) the distribution $J^{r}(\phi)$ of DRS with different $c . \quad c=0$ means a periodic system.
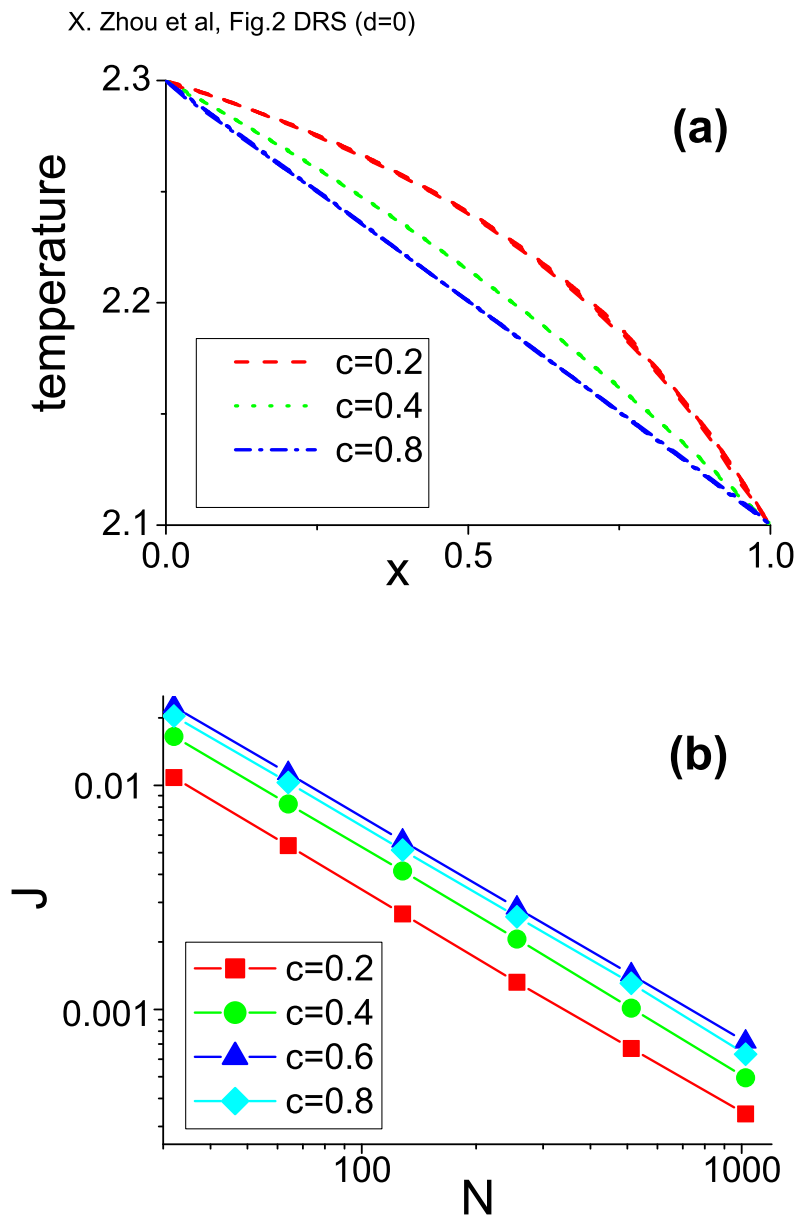

FIG. 2. The results of DRSs with random phase: (a) Temperature profile, where $x=X / N$. For every $c$, we show four temperature profiles, the corresponding chain length are $32,64,128$ and 256, respectively. They are almost identified each other. (b) Heat current $J$ versus $N$. 
X. Zhou et al. Fig. 3, SRS d=0
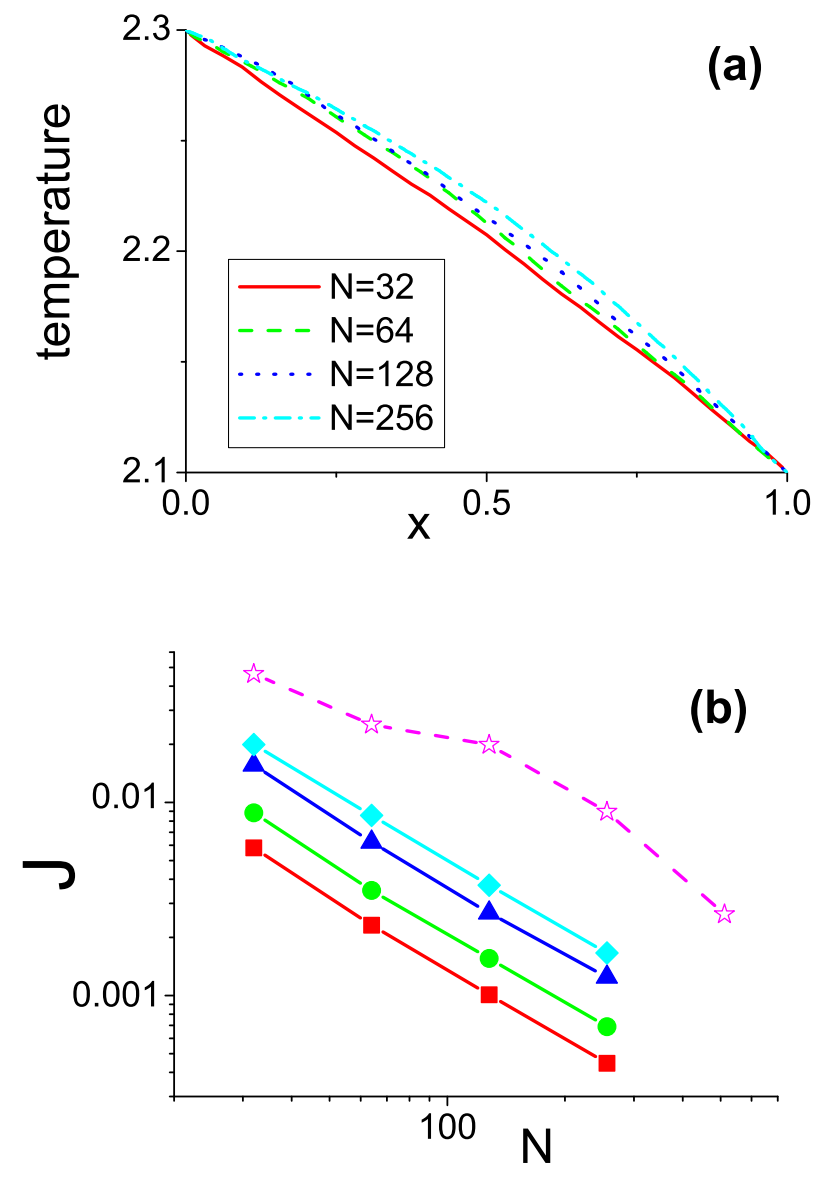

FIG. 3. The results of SRSs with random phase and periodic systems: (a) Temperature profile of four chains, where $c=0.8$. (b) Heat current $J$ versus $N$, from top to bottom, $c$ are 0.0 (periodic system), $0.2,0.4,0.6$ and 0.8 , respectively.


FIG. 4. The correlation of SRSs with random phase: (a) The correlation distribution of different samples, where $N=32$ and $c=0.2$. (b) The fluctuation of correlation $\left\langle D^{2}\right\rangle$ versus $N$. We show the results of three SRSs with different $c$ $(c=0.2,0.5$ and 0.8$)$, they are almost indistinguishable. The solid line is the best fit one. 